\title{
FOURIER INVERSION ON BOREL SUBGROUPS OF CHEVALLEY GROUPS: THE SYMPLECTIC GROUP CASE
}

\author{
BY
}

\author{
RONALD L. LIPSMAN ${ }^{1}$
}

\begin{abstract}
In recent papers, the author and J. A. Wolf have developed the Plancherel theory of parabolic subgroups of real reductive Lie groups. This includes describing the irreducible unitary representations, computing the Plancherel measure, and-since parabolic groups are nonunimodular-explicating the (unbounded) Dixmier-Pukanszky operator that appears in the Plancherel formula. The latter has been discovered to be a special kind of pseudodifferential operator. In this paper, the author considers the problem of extending this analysis to parabolic subgroups of semisimple algebraic groups over an arbitrary local field. Thus far he has restricted his attention to Borel subgroups (i.e. minimal parabolics) in Chevalley groups (i.e. split semisimple groups). The results he has obtained are described in this paper for the case of the symplectic group. The final result is (perhaps surprisingly), to a large extent, independent of the local field over which the group is defined. Another interesting feature of the work is the description of the "pseudodifferential" Dixmier-Pukanszky operator in the nonarchimedean situation.
\end{abstract}

1. Introduction. This paper is another in a series on harmonic analysis of certain nonunimodular locally compact groups. Thus far, this type of analysis has been carried out for some parabolic subgroups of semisimple (or reductive) real Lie groups. The main purpose of this paper is to show how the technique may be extended to semisimple algebraic groups over other local fields.

One of the features that makes harmonic analysis on nonunimodular groups interesting is the Dixmier-Pukanszky operator-i.e. the unbounded positive operator in the Plancherel formula that compensates for lack of unimodularity. In previous work on the subject [2], [5], [7], it has been seen that in the case of parabolic subgroups of semisimple Lie groups these operators turn out to be pseudodifferential operators. More precisely, they are positive powers of the absolute value of certain differential operators. It is therefore not a priori clear what the corresponding objects should be over other local fields. But (in the real case) the operators of ten live on a normal Euclidean subgroup, and so may be analyzed by the Euclidean Fourier transform. Viewed in this way, it is possible to extend the analysis to nonarchimedean fields.

In the context of nonarchimedean fields, I have been mainly interested in Chevalley or split semisimple groups in the four classical categories $A_{n}, B_{n}, C_{n}, D_{n}$.

Received by the editors July 23,1979 and, in revised form, November 20, 1979.

AMS (MOS) subject classifications (1970). Primary 22E50.

${ }^{1}$ Supported in part by NSF under MCS77-01264. 0002-9947/80/0000-0369/\$05.00 
It is a fact of life that only in the $A_{n}$ and $C_{n}$ cases can one expect the DixmierPukanszky operator to live on an abelian subgroup of the nilradical (see $\$ 5$ for a fuller explanation). I have carried out a complete analysis of the Fourier inversioni.e. constructed the irreducible representations and computed the Plancherel measures and the Dixmier-Pukanszky operators-on the Borel subgroup of both $\operatorname{SL}(n, \mathbf{F})$ and $\operatorname{Sp}(n, \mathbf{F})$. The details are quite similar, so I present only those for $\operatorname{Sp}(n, F)$ in what follows. The reader will note that I have placed no restriction on the characteristic of the local field F. Part of the reason for the paper is to illustrate how the Plancherel formula looks basically the same in finite characteristic as it does in characteristic zero.

The paper is organized as follows. In $\$ 2$ we consider the preliminary case of the Borel subgroup of $A_{1}\left(=C_{1}\right)$. We illustrate how the Plancherel formula is quite transparent if we consider the Borel subgroup of GL(2, F) (Prosposition 2.1); but that it becomes more complicated when we pass to $\operatorname{SL}(2, \mathbf{F})$ (Theorem 2.3). The section serves as motivation for the general case, as well as to introduce (as expected, see [5]) the domain problems that arise in harmonic analytic computations with the Dixmier-Pukanszky operator in the nonarchimedean situation. In $\$ 3$ we define a partial Schwartz space and develop some abelian Fourier analysis necessary to overcome the domain problems. The Fourier inversion on the Borel subgroup of $\operatorname{Sp}(n, \mathbf{F})$ is carried out in $\S 4$, the main result being Theorem 4.2. The final section contains some concluding remarks.

Notation. The symbol F denotes a nondiscrete locally compact field. We write $|\cdot|$ to indicate the absolute value on $\mathbf{F}$. In the nonarchimedean case, Haar measure $d x$ on $\mathbf{F}$ is always normalized so that $\int_{|x| \leqslant 1} d x=1$. The Schwartz space $\delta\left(\mathbf{F}^{n}\right)$ is the space of smooth rapidly decreasing-i.e. locally constant and compactly supported when $F$ is nonarchimedean-complex-valued functions on $F^{n}$. If $G$ is a locally compact group we denote by $\delta_{G}$ the modular function of $G$ and by $\hat{G}$ the set of equivalence classes of irreducible unitary representations of $G$. The HilbertSchmidt operators on a Hilbert space $\mathcal{L}$ are denoted $\mathcal{H} \mathcal{S}(\mathcal{E})$ with norm $\|\cdot\|_{2}$. The reader is referred to [3], [4] and [5] for the basic notation and terminology already established in the theory of harmonic analysis on nonunimodular groups. But for the reader's convenience we state a skeletal version of the nonunimodular Plancherel theorem.

TheOREM 1.1. Let $G$ be type I. Then there exist a Dixmier-Pukanszky operator $D$-i.e. a positive selfadjoint operator, semi-invariant of weight $\delta_{G}$ and affiliated with the left ring of $G$-and a positive measure $\mu_{G}$ such that

$$
f\left(1_{G}\right)=\int_{\hat{G}} \operatorname{Tr} \pi(D f) d \mu_{G}(\pi) .
$$

The pair $\left(D, \mu_{G}\right)$ is uniquely determined by equation (1.1).

ACKNOWLEDGEMENT. In the original version of this paper, I separated out the case $\operatorname{ch}(\mathbf{F})=2$ when stating the main results (Proposition 2.2, Theorem 2.3 and Theorem 4.2). The referee suggested the opposite point of view, namely, " . . yes, usually $\mathbf{F}^{*} /\left(\mathbf{F}^{*}\right)^{2}$ is finite; however, the statements and proofs of all theorems 
depend only upon the compactness of $\mathbf{F}^{*} /\left(\mathbf{F}^{*}\right)^{2} \ldots$ it is misleading to overemphasize the case $\operatorname{ch}(\mathbf{F})=2$ since ... [in other cases, other primes]... lead to infinite measure spaces".

It is my pleasure to thank the referee for the valuable suggestions he made. In particular, the statement of Lemma 4.1 was suggested by him to replace a more cumbersome result of my own.

2. The affine motion group: Disintegration of Lebesgue measure on $F$ under the square action of $\mathrm{F}^{*}$. We begin by considering Fourier inversion on the affine motion group over a local field. This section is preliminary and motivational, and is intended to serve the following three purposes: to introduce the technique (in a general sense) for inverting the Fourier transform (i.e. computing the Plancherel formula) on Borel groups; to indicate how the unbounded (Dixmier-Pukanszky) operator arises in the inversion as well as to highlight its properties; and to illustrate the special features of the analysis that appear when $\operatorname{ch}(\mathbf{F}) \neq 0$. For these reasons, the presentation in this section will consist largely of formal computations, with most justifications left for later sections.

Let $\mathbf{F}$ be any nondiscrete locally compact field. We form the locally compact group

$$
\mathcal{Q}(\mathbf{F})=\left\{\left(\begin{array}{ll}
a & b \\
0 & 1
\end{array}\right): a \in \mathbf{F}^{*}, b \in \mathbf{F}\right\}
$$

$\mathcal{Q}(\mathbf{F})$ is not unimodular; indeed it is easy to check that $|a|^{-1} d a d b$ and $|a|^{-2} d a d b$ are right and left Haar measures respectively. Thus the modular function (derivative of right wrt left Haar measure) is $\delta_{\mathscr{Q}(\mathbf{F})}\left(\begin{array}{ll}a & b \\ 0 & 1\end{array}\right)=|a|$. The closed normal subgroup

$$
N=\left\{\left(\begin{array}{ll}
1 & b \\
0 & 1
\end{array}\right): b \in \mathbf{F}\right\}
$$

is regularly embedded and $Q=Q(F)$ is a semidirect product $Q=N H$ where

$$
H=\left\{\left(\begin{array}{ll}
a & 0 \\
0 & 1
\end{array}\right): a \in \mathbf{F}^{*}\right\}
$$

We fix a nontrivial character $\gamma_{1} \in \hat{F}$, normalized so that the pair $\left(\gamma_{1}, d x\right)$ satisfy

$$
\hat{f}(y)=\int_{\mathbf{F}} f(x) \gamma_{1}(x y) d x \Rightarrow f(x)=\int_{\mathbf{F}} \hat{f}(y) \gamma_{1}(-x y) d y, \quad f \in \mathcal{S}(\mathbf{F})
$$

$[1$, p. 141]. Then $\hat{N}$ is identified to $\mathrm{F}$ by

$$
\gamma_{\lambda}\left(\begin{array}{ll}
1 & b \\
0 & 1
\end{array}\right)=\gamma_{\lambda}(b)=\gamma_{1}(\lambda b), \quad \lambda \in \mathbf{F}
$$

$H$ acts on $\hat{N}$ by

$$
h \cdot \gamma_{\lambda}=\gamma_{a^{-1} \lambda} \quad \text { if } h=\left(\begin{array}{ll}
a & 0 \\
0 & 1
\end{array}\right) \in H
$$

and there are two $H$-orbits: $\left\{\gamma_{0}\right\}$ and $\left\{\gamma_{\lambda}: \lambda \in \mathbf{F}^{*}\right\}$. Thus we get two families of inequivalent irreducible unitary representation classes of $\mathcal{Q}$ parameterized by

$$
\chi_{\sigma}\left(\begin{array}{ll}
a & b \\
0 & 1
\end{array}\right)=\sigma(a), \quad \sigma \in\left(\mathbf{F}^{*}\right)^{\wedge}
$$


and the singleton $\pi_{1}=\operatorname{Ind}_{N}^{\mathscr{Q}} \gamma_{1}$. The regular representation of $\mathcal{Q}$ is primary, indeed is an infinite multiple of $\pi_{1}$. Hence the Dixmier-Pukanszky operator for $\mathbb{Q}$ is unique up to a scalar. To see what it must be, we compute $\operatorname{Tr} \pi_{1}(f)$ formally. Naturally we use [3, Theorem 3.2]. Since $\left.\delta_{\mathscr{Q}}\right|_{N} \equiv \delta_{N} \equiv 1$, we obtain

$$
\begin{aligned}
\operatorname{Tr} \pi_{1}(f) & =\int_{N \backslash \mathcal{Q}} \delta_{\mathscr{Q}}(g)^{-1} \int_{n} f\left(g^{-1} n g\right) \gamma_{1}(n) d n d \bar{g} \\
& =\int_{H} \delta_{\mathscr{Q}}(h)^{-1} \int_{N} f\left(h^{-1} n h\right) \gamma_{1}(n) d n d h \\
& =\int_{H} \int_{N} f(n) \gamma_{1}\left(h n h^{-1}\right) d n d h \\
& =\int_{\mathbf{F}^{*}} \int_{\mathbf{F}} f\left(\begin{array}{cc}
1 & \beta \\
0 & 1
\end{array}\right) \gamma_{1}(\alpha \beta) d \beta|\alpha|^{-1} d \alpha \\
& =\int_{\mathbf{F}^{*}} \hat{f}(\alpha)|\alpha|^{-1} d \alpha,
\end{aligned}
$$

where we have set $f(\beta)=f\left(\begin{array}{ll}1 & \beta \\ 0 & 1\end{array}\right)$ and $\hat{f}$ is the Fourier transform (2.1). Since the Dixmier-Pukanszky operator on $\mathcal{Q}$ must satisfy $f\left(1_{\mathscr{Q}}\right)=\operatorname{Tr} \pi_{1}(D f)$, it is clear that we want $D$ to "live on $N$ " and be given there by

$$
(D f)^{\wedge}(\alpha)=|\alpha| \hat{f}(\alpha), \quad f \in \delta(N) .
$$

The splitting $Q=N H$ allows us (see $\$ 3$ for the details) to define from (2.2) an operator $D$ on $\mathbb{Q}$ that is in the left ring. Moreover it is semi-invariant of weight $\delta_{\mathscr{Q}}$. Hence we have (at least formally)

Proposition 2.1 (Plancherel Formula for $Q=Q(F)$ ). We have for suitable $f$, $f\left(1_{\Theta}\right)=\operatorname{Tr} \pi_{1}(D f)$.

The proof is precisely the computation above combined with equation (2.2). By suitable $f$ we mean Schwartz-type functions $f \in \operatorname{Dom} D$ with the property that $D f \in L_{1}$. The question of the "size" of the set of such functions is exactly the kind of domain question encountered previously in [2], [5]. We treat this matter in \$3.

So far we have used the group $A(F)$ to illustrate first how the Plancherel formula is derived and second the nature of the unbounded Dixmier-Pukanszky operator that arises. Next we shall see how finite characteristic of $\mathbf{F}$ can affect the analysis. Consider the Borel subgroup of $\operatorname{GL}(2, F)$ consisting of upper triangular matrices $B=\left\{\left(\begin{array}{ll}a & b \\ 0 & c\end{array}\right): a, c \in \mathbf{F}^{*}, b \in \mathbf{F}\right\}$. The map

$$
\begin{aligned}
& \left(\begin{array}{ll}
a & b \\
0 & c
\end{array}\right)=\left(\begin{array}{ll}
c & 0 \\
0 & c
\end{array}\right)\left(\begin{array}{cc}
a c^{-1} & b c^{-1} \\
0 & 1
\end{array}\right) \rightarrow\left\langle c,\left(\begin{array}{cc}
a c^{-1} & b c^{-1} \\
0 & 1
\end{array}\right)\right\rangle, \\
& B \rightarrow \mathbf{F}^{*} \times \mathbb{Q}(\mathbf{F}),
\end{aligned}
$$

is an isomorphism of topological groups. Hence we can write the Plancherel formula for the Borel group $B$ by invoking Proposition 2.1. However if we consider the group $\operatorname{SL}(2, F)$ instead of $\operatorname{GL}(2, F)$, then the restriction of the above map to $B_{1}=\left\{\left(\begin{array}{cc}a & b \\ 0 & a^{-1}\end{array}\right): a \in \mathbf{F}^{*}, b \in \mathbf{F}\right\}$ fails to be an isomorphism onto $\mathbb{Q}(\mathbf{F})$ exactly when $\left(F^{*}\right)^{2} \varsubsetneqq \mathbf{F}^{*}$. This . s typical of the kind of difficulty one encounters when passing 
from reductive to semisimple groups (over local fields). In particular we shall encounter this situation in $\$ 4$ when we deal with symplectic groups. So we consider separately the locally compact group

$$
\mathscr{B}(\mathbf{F})=\left\{\left(\begin{array}{cc}
a & b \\
0 & a^{-1}
\end{array}\right): a \in \mathbf{F}^{*}, b \in \mathbf{F}\right\} .
$$

$\Re(\mathbf{F})$ is isomorphic to $\mathscr{Q}(\mathbf{F})$ only when $\mathbf{F}=\mathbf{C}$, and then its harmonic analysis is exactly the same as that of $\mathcal{Q}(\mathbf{F})$. When $\mathbf{F}=\mathbf{R}$, the analysis of Proposition 2.1 requires relatively trivial modification; the modification is less trivial when $\operatorname{ch}(\mathbf{F}) \neq$ 0.

The Haar measures on $\mathscr{B}=\mathscr{B}(\mathbf{F})$ are $d a d b$ and $|a|^{-2} d a d b$ respectively; the modular function is $\delta_{\mathfrak{B}}\left(\begin{array}{ll}a & b \\ 0 & a^{-1}\end{array}\right)=|a|^{2}$. In analogy with $\mathbb{Q}$ we describe the representations via the semidirect product $\mathscr{B}=N H$, where

$$
N=\left\{\left(\begin{array}{cc}
1 & b \\
0 & 1
\end{array}\right): b \in \mathbf{F}\right\}, \quad H=\left\{\left(\begin{array}{cc}
a & 0 \\
0 & a^{-1}
\end{array}\right): a \in \mathbf{F}^{*}\right\}
$$

$\hat{N}$ is identified to $\mathrm{F}$ via $\gamma_{1}$ and $H$ acts on $\hat{N}$ by

$$
h \cdot \gamma_{\lambda}=\gamma_{a^{-2} \lambda} \quad \text { if } h=\left(\begin{array}{cc}
a & 0 \\
0 & a^{-1}
\end{array}\right) \in H .
$$

Thus, aside from $\left\{\gamma_{0}\right\}$, there are as many $H$-orbits as there are elements in the group $\mathbf{F}^{*} /\left(\mathbf{F}^{*}\right)^{2}$, where $\left(\mathbf{F}^{*}\right)^{2}=\left\{a^{2}: a \in \mathbf{F}^{*}\right\}$. Let $k_{\mathbf{F}}$ denote the residual field of $\mathbf{F}$. Then it is known that (see [1] or [6])

$$
\left[\mathbf{F}^{*}:\left(\mathbf{F}^{*}\right)^{2}\right]= \begin{cases}1, & \mathbf{F}=\mathbf{C}, \\ 2, & \mathbf{F}=\mathbf{R}, \\ 4, & \operatorname{ch}(\mathbf{F}) \neq 2, \operatorname{ch}\left(k_{\mathbf{F}}\right) \neq 2, \\ \text { power of } 2, & \operatorname{ch}(\mathbf{F}) \neq 2, \operatorname{ch}\left(k_{\mathbf{F}}\right)=2 \\ \infty, & \operatorname{ch}(\mathbf{F})=2\end{cases}
$$

But in all cases $\left(F^{*}\right)^{2}$ is a closed subgroup of $F^{*}$, and the factor group $F^{*} /\left(F^{*}\right)^{2}$ is compact. (This is obvious in all but the last case of (2.3), and in that case is easily verified from the usual realization of F, see [6, II, §3, Proposition 10].) We shall denote by $s: \mathbf{F}^{*} /\left(\mathbf{F}^{*}\right)^{2} \rightarrow \mathbf{F}^{*}$ a Borel cross-section. For convenience we write $R=R_{\mathrm{F}}=\mathrm{F}^{*} /\left(\mathrm{F}^{*}\right)^{2}$. The stability group for a nontrivial character $\gamma_{\lambda}$ is

$$
H_{\lambda}=\left\{h \in H: h \cdot \gamma_{\lambda}=\gamma_{\lambda}\right\}=Z_{\mathbf{F}}=\left\{\left(\begin{array}{ll}
\varepsilon & 0 \\
0 & \varepsilon
\end{array}\right): \varepsilon= \pm 1_{\mathbf{F}}\right\} \text {. }
$$

$Z=Z_{\mathbf{F}}$ contains 2 elements except when $\operatorname{ch}(\mathbf{F})=2$. Then the representations of $\mathscr{B}$ fall into two classes.

$$
\begin{gathered}
\left\{\chi_{\sigma}: \sigma \in\left(\mathrm{F}^{*}\right)^{\wedge}\right\}, \quad \chi_{0}\left(\begin{array}{cc}
a & b \\
0 & a^{-1}
\end{array}\right)=\sigma(a), \\
\pi_{\rho, \eta}=\operatorname{Ind}_{N Z}^{\mathscr{B}} \gamma_{s(\rho)} \times \eta, \quad \eta \in \hat{Z}, \rho \in R .
\end{gathered}
$$

Now as indicated in the subtitle of this section, we perform a special disintegration. 
Proposition 2.2. We have

$$
\int_{\mathbf{F}} f(x) d x=\int_{R_{\mathbf{F}}} \int_{\mathbf{F}^{*}} f\left(a^{2 \zeta}\right)|a| d a|s(\zeta)| d \zeta, \quad f \in \mathcal{S}\left(\mathbf{F}^{*}\right)
$$

where dל is the Haar measure on $R_{\mathbf{F}}=\mathbf{F}^{*} /\left(\mathbf{F}^{*}\right)^{2}$ uniquely determined by

$$
\int_{\mathbf{F}^{*}} f(y) \frac{d y}{|y|}=\int_{R_{\mathbf{F}}} \int_{\left(\mathbf{F}^{*}\right)^{2}} f(z \zeta) d z d \zeta
$$

and $d z$ is the Haar measure on $\left(\mathbf{F}^{*}\right)^{2}$ given by $f \rightarrow \int f\left(a^{2}\right) d a /|a|$.

Proof. Lebesgue measure $d x$ on $\mathbf{F}$ is relatively invariant under the action of $\mathbf{F}^{*}$ (given by $a \cdot x=a^{2} x$ ) with modulus $|a|^{2}$. But every nonzero orbit carries a well-determined measure which is relatively invariant of the same modulus, namely $f \rightarrow \int_{\mathbf{F}^{*}} f\left(a^{2} s(\rho)\right)|a| d a$. Hence (by [3, Theorem 2.1]) there is a unique pseudo-image $d \bar{x}$ of $\left.d x\right|_{F^{*}}$ on $R_{\mathbf{F}}$ such that

$$
\int_{\mathbf{F}^{*}} f(x) d x=\int_{R_{\mathbf{F}}} \int_{\mathbf{F}^{*}} f(a \cdot s(\bar{x}))|a| d a d \bar{x}
$$

In order to compute $d \bar{x}$, we can reason as follows. If $f \in \delta\left(F^{*}\right)$, then $g(x)=$ $|x| f(x) \in \mathcal{S}(\mathbf{F})$ and we have

$$
\begin{aligned}
\int_{\mathbf{F}} f(x) d x & =\int_{\mathbf{F}^{*}} g(x) \frac{d x}{|x|}=\int_{\mathbf{F}^{*} /\left(\mathbf{F}^{*}\right)^{2}} \int_{\left(\mathbf{F}^{*}\right)^{2}} g(u \zeta) d u d \zeta \\
& =\int_{\mathbf{F}^{*} /\left(\mathbf{F}^{*}\right)^{2}} \int_{\mathbf{F}^{*}} g\left(a^{2} s(\rho)\right) \frac{d a}{|a|} d \zeta \\
& =\int_{\mathbf{F}^{*} /\left(\mathbf{F}^{*}\right)^{2}} \int_{\mathbf{F}^{*}} f\left(a^{2} \zeta\right)|a||s(\zeta)| d a d \zeta
\end{aligned}
$$

Thus $|s(\zeta)| d \zeta$ is the unique pseudo-image of $d x$ in $R_{\mathbf{F}}$ determined by the choices of $d x$ on $\mathbf{F}$ and $f\left(a^{2}\right) d a /|a|$ on $\left(\mathbf{F}^{*}\right)^{2}$.

Before continuing we observe that $d \zeta$ is not normalized Haar measure on $\boldsymbol{R}_{\mathbf{F}}$. In fact it is easy to see that in case $\operatorname{ch}(\mathbf{F}) \neq 2$, the total mass assigned to $R_{F}$ by $d \zeta$ is 2 .

Now we can give the Fourier inversion on $\mathscr{B}(\mathbf{F})$.

Theorem 2.3 (Plancherel Formula for $\mathscr{B}=\mathscr{B}(\mathbf{F})$ ). With $D$ defined by formula (2.2) we have

$$
f\left(1_{\mathscr{B}}\right)=\sum_{\hat{Z}} \int_{R_{\mathbf{F}}} \operatorname{Tr} \pi_{\rho, \eta}(D f) d \rho
$$

Proof. Before giving the computation, we note that the final result is independent of the section $s: R_{\mathbf{F}} \rightarrow \mathbf{F}^{*}$. As with Proposition 2.1 , we supply only the formal computations here. The complete justification relies on results of the next section. We employ the usual tool, i.e. [3, Theorem 3.2]. 


$$
\begin{aligned}
\operatorname{Tr} \pi_{\rho, \eta}(f) & =\int_{N Z \backslash \mathscr{B}} \delta(g)^{-1} \sum_{Z} \int_{N} f\left(g^{-1} n z g\right) \gamma_{s(\rho)}(n) \eta(z) d n d \bar{g} \\
& =\int_{Z \backslash H} \delta(h)^{-1} \sum_{Z} \int_{N} f\left(h^{-1} n z h\right) \gamma_{s(\rho)}(n) \eta(z) d n d h \\
& =\int_{Z \backslash H} \sum_{Z} \int_{N} f(n z) \gamma_{s(\rho)}\left(h n h^{-1}\right) \eta(z) d n d h
\end{aligned}
$$

Therefore

$$
\begin{aligned}
\sum_{\hat{Z}} \int_{R_{\mathbf{F}}} & \operatorname{Tr} \pi_{\rho, n}(D f) d \rho \\
& =\sum_{\hat{Z}} \int_{R_{\mathbf{F}}} \int_{Z \backslash H} \sum_{Z} \int_{N} D f(n z) \gamma_{s(\rho)}\left(h n h^{-1}\right) \eta(z) d n d h d \rho \\
& =2 \int_{R_{\mathbf{F}}} \int_{Z \backslash H} \int_{n} D f(n) \gamma_{s(\rho)}\left(h n h^{-1}\right) d n d h d \rho \\
& =\int_{R_{\mathbf{F}}} \int_{H} \int_{N} D f(n) \gamma_{s(\rho)}\left(h n h^{-1}\right) d n d h d \rho \\
& =\int_{R_{\mathbf{F}}} \int_{F^{*}} \int_{\mathbf{F}} D f\left(\begin{array}{ll}
1 & \beta \\
0 & 1
\end{array}\right) \gamma_{s(\rho)}\left(\alpha^{2} \beta\right) d \beta|\alpha|^{-1} d \alpha d \rho \\
& =\int_{R_{\mathbf{F}}} \int_{\mathbf{F}^{*}}(D f)^{-}\left(\alpha^{2} s(\rho)\right)|\alpha|^{-1} d \alpha d \rho \\
& =\int_{R_{\mathbf{F}}} \int_{\mathbf{F}^{*}} \hat{f}\left(\alpha^{2} s(\rho)\right)|\alpha||s(\rho)| d \alpha d \rho \\
& =\int_{\mathbf{F}} \hat{f}(x) d x \quad \text { by Proposition } 2.2 \\
& =f(0)=f\left(1_{\mathscr{B}}\right) .
\end{aligned}
$$

In (2.4) both the ' $Z$ ' and the ' 2 ' are omitted if $\operatorname{ch}(\mathbf{F})=2$.

3. The unbounded operators. In this section we define precisely the unbounded operators that appear in the Plancherel formulae of $\$ \S 2$ and 4. The material here is quite analogous to that in $[5, \S \S 1,3]$, and therefore we shall not provide all details. The basic need is to define and analyze the analog of the partial Schwartz space of $[5, \S 3 \mathrm{~b}]$. The reader is referred to [5] for any undefined notation or terminology.

Let $\mathbf{F}$ be a nondiscrete local field. By an F-manifold we shall mean the F-rational points $V=V(F)$ of an affine algebraic variety $\mathbf{V}$ def $/ F$. If $V$ is an affine algebraic group, $V$ is called an F-group. We shall be dealing with $V$ of the form $V=Z \times W$, where $W$ is an F-manifold and $Z$ is an F-group that is unipotent abelian, i.e. $Z$ is a connected unipotent abelian algebraic group def $/ \mathbf{F}$. In addition we shall assume that we have a positive Radon measure $d v=d z d w$, where $d z$ is Haar measure on $Z$. We fix a nontrivial character $\gamma_{1} \in \hat{\mathbf{F}}$ (as in $\$ 2$ ), as well as a nondegenerate F-bilinear form $(\cdot, \cdot)$ on $Z \cong \mathbf{F}^{k}, k=\operatorname{dim}_{\mathbf{F}} Z$. Then we have a partial Fourier 
transform

$$
\hat{f}(\xi, w)=\mathscr{F} f(\xi, w)=\int_{Z} f(z, w) \gamma_{1}(z, \xi) d z, \quad \xi \in Z, w \in W .
$$

Suppose that $\psi$ is an F-valued polynomial function on $Z$. Then for any $t \geqslant 0$ we can define an operator $|\Psi|^{t}$ by

$$
\mathscr{F}\left(|\Psi|^{t} f\right)(\xi, w)=|\psi(\xi)|^{t} \mathscr{F}(f)(\xi, w) .
$$

As an operator on $L_{2}(V, d v),|\Psi|^{t}$ is symmetric with positive selfadjoint closure.

Next we want to specify a partial Schwartz space on $V$ (as in $[5, \S 3 b])$. The primary requirements for this space are that it should be large (dense in $L_{2}(V)$ ), contained in $\operatorname{Dom}|\Psi|^{t}$ for any $t \geqslant 0$, and be moved by $|\Psi|^{t}$ into integrable functions. Here is the space.

Definition 3.1. Let $p$ be an F-valued polynomial function on $Z$. Set $\delta_{p}(V)=$ $\{f: V \rightarrow \mathbf{C}$ having the following properties (i)-(iv) $\}$.

(i) $f$ is smooth (i.e. $C^{\infty}$ if $\mathbf{F}$ is archimedean, locally constant if $\mathbf{F}$ is nonarchimedean);

(ii) $\exists L \subseteq W$ compact such that $f(z, w)=0$ if $w \notin L$;

(iii)(a) (F archimedean) $\forall$ polynomial $q(z)$ and $\forall$ constant coefficient differential operator $T$ on $Z$, $\sup _{z, w}|q(z) T f(z, w)|<\infty$;

(iii)(b) (F nonarchimedean) $f$ is compactly supported;

(iv) $\exists \Re$ neighborhood of $\{\xi \in Z: p(\xi)=0\}$ such that $\mathscr{F}(f)(\xi, w)=0$ if $\xi \in \Re$. Here is the main result of this section.

Proposition 3.2. Let $\psi$ be an $\mathbf{F}$-valued polynomial function on $Z$. Then

(i) $\delta_{\psi}(V)$ is dense in $L_{2}(V, d v)$.

(ii) $\delta_{\psi}(V) \subseteq \operatorname{Dom}|\Psi|^{t}, \forall t \geqslant 0$.

(iii) $|\Psi|^{t} \mathcal{S}_{\psi}(V) \subseteq L_{1}(V, d v), \forall t \geqslant 0$.

(iv) Let $V=Z W$ be an F-group with $Z$ normal and $d v=d z d w$ a right Haar measure. Then

(a) Dom $|\Psi|^{t} \cap|\Psi|^{-t} L_{1}(V) \cap L_{2}(V)^{\mathcal{L}}$ is dense in $L_{2}(V),\left(L_{2}(V)^{\mathfrak{L}}=\right.$ left bounded elements in $L_{2}$ ),

(b) if $f \in \mathcal{S}_{\psi}(V)$, then $f *{ }_{V} f^{*} \in \mathcal{S}_{\psi}(V)$,

(c) $|\Psi|^{t}$ is affiliated with the left ring of $V$,

(d) if $|\psi|^{t}$ is semi-invariant of weight $\Delta: V \rightarrow \mathbf{R}_{+}^{*}$, then $|\Psi|^{t}$ has the same property, i.e. if $|\psi(\tilde{v} \cdot \xi)|^{t}=\Delta(v)|\psi(\xi)|^{t}$ (where $\tilde{v} \cdot$ denotes the transpose action of $V$ on $Z$ determined by $(\cdot, \cdot))$, then $\operatorname{Ad}(v)|\Psi|^{t}=\Delta(v)|\Psi|^{t}$.

Proof. Much of the argument is very similar to the corresponding parts of $[5$, Lemma 3.10 and Corollary 3.11].

(i) If we let $\delta(V)$ denote the set of functions on $V$ having properties (i)-(iii) of Definition 3.1, then the partial Fourier transform is a linear (topological) isomorphism of $\delta(V)$ onto itself. Moreover $\delta(V)$ is dense in $L_{p}(V), 1<p<\infty$. Reasoning exactly as in [5, Proof of Lemma 3.10], we see easily that the proof reduces to the case $V=Z$. Then it follows from the fact that the space $\{f \in$ $\mathcal{S}(Z): f=0$ near $\left.\psi^{-1}(0)\right\}$ is dense in $L_{2}(Z)$. 
(ii) Since $\operatorname{Dom}|\Psi|^{t}=\left\{f \in L_{2}(V):|\psi(\xi)|^{t} \hat{f}(\xi, w) \in L_{2}(V)\right\}$, it is obvious that $\mathcal{S}_{\psi}(V) \subseteq \operatorname{Dom}|\Psi|^{t}$.

(iii) Clearly $|\Psi|^{t} f(z, w)=\mathscr{F}^{-1}\left\{|\psi(\xi)|^{t} \mathscr{F}(f)\right\}(z, w), f \in \mathcal{\delta}_{\psi}(V)$. Hence $|\Psi|^{t} \delta_{\psi}(V) \subseteq$ $\delta(V) \subseteq L_{1}(V, d v)$.

(iv) Now we assume (as will be the case in $\S 4$ ) that $V=Z W$ is an F-group, and that $Z$ is unipotent, abelian and normal.

(a) This is true because

$$
\mathcal{\delta}_{\psi}(V) \subseteq \operatorname{Dom}|\Psi|^{t} \cap|\Psi|^{-t} L_{1}(V) \cap L_{2}(V)^{\mathfrak{L}},
$$

and incidentally insures that $[5$, Proposition 1.6] is valid.

(b) In the archimedean case, the proof is exactly the same as that of [5, Corollary 3.11(3)]. In the nonarchimedean case, the portion that must be altered is that corresponding to property (iii) of Definition 3.1; but the proof is even easier in that situation.

(c) This computation is straightforward. If $v \in V$, we write $v=z_{v} w_{v} \in Z W$ and $f(v)=f(z, w)$. Then

$$
\left(f^{v}\right)^{\wedge}(\xi, w)=\int_{Z} \gamma_{1}(\xi, u) f(u w v) d u=\gamma_{1}\left(\xi, z_{w v}^{-1}\right) \hat{f}\left(\xi, w_{w v}\right) .
$$

Hence, setting $D=|\Psi|^{t}$, we see that

$$
\left(D\left(f^{v}\right)\right)^{\wedge}(\xi, w)=|\psi(\xi)|^{t}\left(f^{v}\right)^{\wedge}(\xi, w)=|\psi(\xi)|^{t} \gamma_{1}\left(\xi, z_{w v}^{-1}\right) \hat{f}\left(\xi, w_{w v}\right)
$$

and

$$
\left((D f)^{v}\right)^{\wedge}(\xi, w)=\gamma_{1}\left(\xi, z_{w v}^{-1}\right)(D f)^{\wedge}\left(\xi, w_{w v}\right)=\gamma_{1}\left(\xi, z_{w v}^{-1}\right)|\psi(\xi)|^{t} \hat{f}\left(\xi, w_{w v}\right)
$$

Thus $D$ is affiliated with the left ring.

(d) This time we translate on the left. Writing $v \cdot u=v u v^{-1}$, we have

$$
\begin{aligned}
\left(f_{v}\right)^{\wedge}(\xi, w) & =\int \gamma_{1}(\xi, u) f\left(v^{-1} u w\right) d u=\int \gamma_{1}(\xi, u) f\left(v^{-1} \cdot u z_{v^{-1} w} w_{v^{-1} w}\right) d u \\
& =\delta_{Z}(v) \int \gamma_{1}(\xi, v \cdot u) f\left(u z_{v^{-1} w} w_{v^{-1} w}\right) d u \\
& =\delta_{Z}(v) \int \gamma_{1}\left(\xi, v \cdot\left(u z_{v^{-1} w}^{-1}\right)\right) f\left(u w_{v^{-1} w}\right) d u \\
& =\delta_{Z}(v) \gamma_{1}\left(\tilde{v} \cdot \xi, z_{v^{-1} w}^{-1}\right) \hat{f}\left(\tilde{v} \cdot \xi, w_{v^{-1} w}\right) .
\end{aligned}
$$

Therefore, using $D=|\Psi|^{t}$ as above, we have

$$
(D f)_{v}^{\hat{v}}(\xi, w)=\delta_{Z}(v) \gamma_{1}\left(\tilde{v} \cdot \xi, z_{v^{-1} w}^{-1}\right)|\psi(\tilde{v} \cdot \xi)|^{t} \hat{f}\left(\tilde{v} \cdot \xi, w_{v^{-1} w}\right)
$$

and

$$
D\left(f_{v}\right)^{\wedge}(\xi, w)=|\psi(\xi)|^{t}\left(f_{v}\right)^{\wedge}(\xi, w)=|\psi(\xi)|^{t} \delta_{Z}(v) \gamma_{1}\left(\tilde{v} \cdot \xi, z_{v^{-1} w}^{-1}\right) \hat{f}\left(\tilde{v} \cdot \xi, w_{v^{-1} w}\right) .
$$

Hence $(D f)_{v}=\Delta(v) D\left(f_{v}\right)$. This completes the proof.

REMARKS. 1. In [2], [5] we interpreted $|\Psi|^{t}$ as a positive power of the absolute value of the constant coefficient differential operator $\Psi=\hat{\psi}$. This is a special kind of pseudodifferential operator. In the nonarchimedean case $\hat{\psi}$ makes no sense as a differential operator. But constant coefficient differential operators can be identified to convolutions with distributions supported at $\{0\}$. It is thought by some that 
the "correct" nonarchimedean analog is convolution by distributions of compact support. Of course $|\psi|^{t}$ is a distribution on $Z$, and $|\Psi|^{t}$ is convolution by its Fourier transform. Sometimes these Fourier transforms can be identified as regularizations of distributions given by explicit functions. For example on $V=\mathbf{F}$, the operator $(D f)^{\wedge}(x)=|x| \hat{f}(x)$ is precisely convolution by the distribution $1 /|x|^{2}$ regularized as in [1, Chapter 2, §2].

2. Regarding the operator $D$ of $\S 2$, we observe that the corresponding polynomial on $V=N \cong \mathbf{F}$ is $\psi\left(\begin{array}{ll}1 & \beta \\ 0 & 1\end{array}\right)=\beta$, and

$$
\mathcal{S}_{\psi}(G)=\{f \in \mathcal{S}(G): \hat{f}=0 \text { near } 0\}, \quad G=\mathscr{Q} \text { or } \mathscr{B} .
$$

The Plancherel formulae of Proposition 2.1 and Theorem 2.3 are valid for $f \in \mathcal{S}_{\psi}$.

3. Wolf has pointed out to me that C. W. Onneweer has developed a theory of differentiation in the nonarchimedean case. I do not know if it is relevant to these matters.

4. In the applications of Proposition 3.2 in $\S 4, W$ will always be a group, but I envision a situation (as in [5]) where that may not be the case.

4. Fourier transform on the Borel subgroup of the symplectic group. If $\mathfrak{T}$ denotes a $2 n$-dimensional vector space over $\mathbf{F}$ on which there is a symplectic form $\langle\cdot, \cdot\rangle$, then we write $\operatorname{Sp}(\mathfrak{V})$ for the group of $\mathbf{F}$-linear transformations of $\mathfrak{V}$ that preserve the form. For example on $\mathbf{F}^{2 n}=\left\{\left(x_{1}, \ldots, x_{n} ; y_{1}, \ldots, y_{n}\right): x_{i}, y_{i} \in \mathbf{F}\right\}$ we may take

$$
\left\langle\left(x^{1}, y^{1}\right),\left(x^{2}, y^{2}\right)\right\rangle=\sum_{1}^{n}\left(x_{i}^{1} y_{i}^{2}-x_{i}^{2} y_{i}^{1}\right) .
$$

Then $\operatorname{Sp}(n, F)$ is the subgroup of $\operatorname{GL}(2 n, \mathbf{F})$ that preserves $\langle\cdot, \cdot\rangle$. Let us denote by $G=G_{n, \mathbf{F}}$ a Borel subgroup of $\operatorname{Sp}(n, \mathbf{F})$. Then it is known that $G$ can be realized as a semidirect product $G=N A$ where

$$
A=\left\{\left(\begin{array}{cc}
\Gamma & 0 \\
0 & \Gamma^{-1}
\end{array}\right): \Gamma \in \mathrm{GL}(n, \mathbf{F}) \text { is diagonal }\right\},
$$

and the normal subgroup $N$ is itself a semidirect product $N=U V$ with

$$
\begin{aligned}
& U=\left\{\left(\begin{array}{cc}
I & B \\
0 & I
\end{array}\right): B \in \mathbf{F}^{n \times n}, B={ }^{t} B \text { is symmetric }\right\} \\
& V=\left\{\left(\begin{array}{cc}
X & 0 \\
0 & { }^{t} X^{-1}
\end{array}\right): X \in \mathrm{GL}(n, \mathbf{F}) \text { is lower triangular and unipotent }\right\} .
\end{aligned}
$$

The group action is of course matrix multiplication and $U$ is abelian normal in $G$. We shall describe the representation theory and Plancherel formula of $G$ via the group extension $U \triangleleft G$. Thus we write $G=U M$ where

$$
M=V A=\left\{\left(\begin{array}{cc}
W & 0 \\
0 & { }^{t} W^{-1}
\end{array}\right): W \in \mathrm{GL}(n, \mathbf{F}) \text { is lower triangular }\right\} .
$$

We shall make use of the following easily-verified linear algebra result.

LeMmA 4.1. Let $B={ }^{t} B \in \mathrm{F}^{n \times n}$. Let $\Delta_{k}(B)$ be the determinant of the lower right $k \times k$ minor of $B, k=1,2, \ldots, n$. Assume $\Delta_{k}(B) \neq 0, k=1, \ldots, n$. Then there exists a unique $n \times n$ lower triangular unipotent matrix $X$ such that ${ }^{\prime} X B X$ is diagonal. 
Let us set

$$
\mathscr{Q}=\left\{u \in U=\left(\begin{array}{cc}
I & B \\
0 & I
\end{array}\right): \Delta_{k}(B) \neq 0, k=1, \ldots, n\right\} .
$$

It is clear that $\mathscr{U}$ is a Zariski-open, $M$-invariant subset of $U$. Furthermore we obtain easily from Lemma 4.1 that

$$
\begin{aligned}
V \times \mathcal{U}_{d} & \rightarrow \mathscr{Q}\left(\mathcal{U}_{d}=\left\{u=\left(\begin{array}{ll}
I & B \\
0 & I
\end{array}\right) \in \mathcal{U}: B \text { diagonal }\right\}\right), \\
(X, \Gamma) & \rightarrow{ }^{t} X \Gamma X
\end{aligned}
$$

is a bijection. It follows immediately that $\mathscr{Q} / M=\bigcup_{d} / A$. This enables us to write down an explicit cross-section of $\mathcal{U} / \boldsymbol{M}$.

Let $s: \mathbf{F}^{*} /\left(\mathbf{F}^{*}\right)^{2} \rightarrow \mathbf{F}^{*}$ be a Borel cross-section as in $\S 2$. Put

$$
\rho=\left(\rho_{1}, \ldots, \rho_{n}\right) \in R_{\mathrm{F}} \times \cdots \times R_{\mathrm{F}}=R_{\mathrm{F}}^{n}
$$

and define $\gamma_{\rho}$ to be the diagonal matrix with entries $\left(\gamma_{\rho}\right)_{k k}=s\left(\rho_{n-k+1}\right) s\left(\rho_{n-k}\right)$, $k=1, \ldots, n$; it being understood that $s\left(\rho_{0}\right) \equiv 1$. That is

$$
\gamma_{\rho}=\left(\begin{array}{cccc}
s\left(\rho_{n}\right) s\left(\rho_{n-1}\right)^{-1} & & & 0 \\
& s\left(\rho_{n-1}\right) s\left(\rho_{n-2}\right)^{-1} & & \\
& \ddots & s\left(\rho_{2}\right) s\left(\rho_{1}\right)^{-1} & \\
0 & & & s\left(\rho_{1}\right)
\end{array}\right) .
$$

Then it is clear that $\mathcal{C}=\bigodot_{n}=\left\{\gamma_{\rho}: \rho \in R_{\mathrm{F}}^{n}\right\}$ is a Borel cross-section for $\mathcal{U} / M$.

As usual we fix a nontrivial unitary charcter of $F$, but henceforth we denote it by $\chi_{1}$. Then $U$ is self-dual, and for any symmetric $n \times n$ matrix $\gamma$ we identify it to the element of $\hat{U}$ defined by

$$
\gamma\left(\begin{array}{ll}
I & B \\
0 & I
\end{array}\right)=\chi_{1}(\operatorname{Tr} \gamma B)
$$

The action of $M$ on $\hat{U}$ is

$$
\begin{aligned}
(m \cdot \gamma)(u) & =\gamma\left(m^{-1} u m\right)=\gamma\left[\left(\begin{array}{cc}
W^{-1} & 0 \\
0 & { }^{t} W
\end{array}\right)\left(\begin{array}{cc}
I & B \\
0 & I
\end{array}\right)\left(\begin{array}{cc}
W & 0 \\
0 & { }^{t} W^{-1}
\end{array}\right)\right] \\
& =\gamma\left(\begin{array}{cc}
I & W^{-1} B^{t} W^{-1} \\
0 & I
\end{array}\right) \\
& =\chi_{1}\left(\operatorname{Tr} \gamma W^{-1} B^{t} W^{-1}\right)=\chi_{1}\left(\operatorname{Tr}^{t} W^{-1} \gamma W^{-1} B\right)
\end{aligned}
$$

that is

$$
m \cdot \gamma={ }^{t} W^{-1} \gamma W^{-1} \quad \text { if } m=\left(\begin{array}{cc}
W & 0 \\
0 & { }^{t} W^{-1}
\end{array}\right) \in M
$$


Now it is fairly routine to see that the stability group for the action of $M$ on any $\gamma_{\rho} \in \mathcal{C}$ is

$$
Z=\left\{\left(\begin{array}{ll}
\delta & 0 \\
0 & \delta
\end{array}\right): \delta=\left(\begin{array}{lll}
\delta_{1} & & 0 \\
& \ddots & \\
0 & & \delta_{n}
\end{array}\right), \delta_{i}^{2}=1\right\} \cong\left(Z_{2}\right)^{n} .
$$

Thus the generic irreducible representations of $G$ are parameterized by

$$
\pi(\rho, \eta)=\operatorname{Ind}_{U Z}^{G} \gamma_{\rho} \times \eta, \quad \rho \in R_{\mathrm{F}}^{n}, \eta \in \hat{Z} .
$$

In case $\operatorname{ch}(\mathbf{F})=2, Z$ is trivial. It will be convenient later to work with

$$
\pi(\rho)=\operatorname{Ind}_{U}^{G} \gamma_{\rho} \cong \sum_{\eta \in \hat{Z}}^{\oplus} \pi(\rho, n), \quad \rho \in R_{\mathrm{F}}^{n}
$$

Note that (as in $\S 2$ ) the regular representation of $G$ is a direct sum of irreducibles exactly when $\operatorname{ch}(\mathbf{F}) \neq 2$.

Next we enumerate some Haar measures and modular functions in terms of the following coordinates. $G=U M$ where

$$
\begin{gathered}
M=\left(\begin{array}{cc}
W & 0 \\
0 & { }^{t} W^{-1}
\end{array}\right), \quad W=\left(\begin{array}{lll}
\alpha_{1} & & 0 \\
& \ddots & \\
w_{i j} & \alpha_{n}
\end{array}\right), \quad \alpha_{i} \in \mathbf{F}^{*}, w_{i j} \in \mathbf{F}, \\
U=\left(\begin{array}{cc}
I & B \\
0 & I
\end{array}\right), \quad B=\left(u_{i j}\right), \quad u_{i j}=u_{j i} \in \mathbf{F} .
\end{gathered}
$$

Then

$$
\begin{gathered}
d u=\prod_{1<i<j<n} d u_{i j} \text { is Haar measure on } U, \\
\prod_{1<i<j<n} d w_{i j} \frac{d \alpha_{1}}{\left|\alpha_{1}\right|^{n}} \cdots \frac{d \alpha_{n}}{\left|\alpha_{n}\right|} \text { is right Haar measure on } M \text { and } \\
\delta_{G}(u m)=\left|\alpha_{1}\right|^{2}\left|\alpha_{2}\right|^{4} \cdots\left|\alpha_{n}\right|^{2 n}, \quad \delta_{M}(m)=\left|\alpha_{1}\right|^{-(n-1)} \cdots\left|\alpha_{n}\right|^{n-1} .
\end{gathered}
$$

We are now ready to define the Dixmier-Pukanszky operator. The group $G$ is an F-manifold (as explained in \$3) of the form $G=U S \approx U \times S$. The DixmierPukanszky operator "lives on $U$ ", and is determined according to the scheme of that section by the polynomial $\psi(\gamma)=\prod_{k=1}^{n} \Delta_{k}(\gamma)$. That is we define

$$
(D f)^{\wedge}(\gamma, m)=\left|\prod_{k=1}^{n} \Delta_{k}(\gamma)\right| \hat{f}(\gamma, m), \quad \gamma \in \hat{U}, f \in \mathcal{\delta}_{\psi}(G),
$$

where

$$
\hat{f}(\gamma, m)=\int_{U} \chi_{1}(\operatorname{Tr} \gamma B) f(u m) d u, \quad u=\left(\begin{array}{ll}
I & B \\
0 & I
\end{array}\right) .
$$

According to Proposition 3.2, $D$ has all the requisite properties and is semi-invariant of weight $\delta_{G}$, since $\psi(\gamma)$ is semi-invariant under the action of $M$ with weight $\alpha_{1}^{2} \alpha_{2}^{4} \cdots \alpha_{n}^{2 n}$. We can now state the main result. 
Theorem 4.2 (Plancherel Formula for $G=G_{n, F}$ ). With $D$ defined by equation (4.2), we have

$$
f\left(1_{G}\right)=\sum_{\hat{Z}} \int_{R_{F}^{n}} \operatorname{Tr} \pi(\rho, \eta)(D F) d \rho, \quad f \in \delta(G),
$$

where $d \rho=d \rho_{1} \cdots d \rho_{n}$ and each $d \rho_{i}$ is the same as $d \zeta$ in Proposition 2.2.

Proof. We shall also use the results of $[5, \S 1]$. Because of $[5$, Theorem 1.1, Proposition 1.6] and Proposition 3.2, we see it is enough to prove

$$
\int_{G}\left|f_{1}(g)\right|^{2} d g=\sum_{\hat{z}} \int_{R_{F}^{n}}\left\|\pi(\rho, n)\left(D^{1 / 2} f_{1}\right)\right\|_{2}^{2} d \rho, \quad f_{1} \in \mathcal{S}_{\psi}(G) .
$$

Set $D^{\prime}=\Lambda^{-2} D$, where $\left(\Lambda^{-2} f\right)(g)=\delta_{G}(g) f(g)$ (see [5, \&1] and [3], [4]). $D^{\prime}$ is affiliated with the right ring of $G$. Since $\delta_{G}$ lives on $M$, it is clear that Proposition 3.2 is also valid for $D^{\prime}$. Put $f_{2}=f_{1} * f_{1}^{*}$. By Proposition 3.2, $f_{2} \in \delta_{\psi}(G)$. Then

$$
\begin{aligned}
\left\|\pi(\rho, \eta)\left(D^{1 / 2} f_{1}\right)\right\|_{2}^{2} & =\operatorname{Tr}\left\{\pi(\rho, \eta)\left(D^{1 / 2} f_{1}\right)\left[\pi(\rho, \eta)\left(D^{1 / 2} f_{1}\right)\right]^{*}\right\} \\
& =\operatorname{Tr} \pi(\rho, \eta)\left(D^{1 / 2} f_{1} * D^{1 / 2} f_{1}^{*}\right) \\
& =\operatorname{Tr} \pi(\rho, \eta)\left(D^{1 / 2} D^{1 / 2} f_{2}\right) .
\end{aligned}
$$

Thus we need to prove

$$
f_{2}\left(1_{G}\right)=\sum_{\hat{Z}} \int_{R_{\hat{F}}^{n}} \operatorname{Tr} \pi(\rho, \eta)\left(D^{1 / 2} D^{\prime 1 / 2} f_{2}\right) d \rho .
$$

Note that $D^{1 / 2} D^{1 / 2} f_{2}$ is a function to which [3, Theorem 3.2] applies. (It is a convolution of an $L_{1}$ function with its adjoint.) Now set $f=\Lambda^{-1} f_{2} \in \delta_{\psi}(G)$. Substituting, we see that it is enough to prove (4.3) under the assumptions: $f \in \mathcal{S}_{\psi}(G), D f \in L_{1}(G)$ and $D f$ is a function to which [3, Theorem 3.2] applies.

The remainder of the argument is computational. We first observe that it is enough-by equation (4.1) and the last equation-to prove

$$
f\left(1_{G}\right)=\int_{R_{F}^{n}} \operatorname{Tr} \pi(\rho)(D f) d \rho
$$

So we utilize [3, Theorem 3.2]:

$$
\begin{aligned}
\operatorname{Tr} \pi(\rho)(D f) & =\operatorname{Tr} \operatorname{Ind}_{U}^{G} \gamma_{\rho}(D f) \\
& =\int_{M} \delta_{G}(m)^{-1} \int_{U} D f\left(m^{-1} u m\right) \gamma_{\rho}(u) d u d m \\
& =\int_{M} \delta_{M}(m)^{-1} \int_{U} D f(u) \gamma_{\rho}\left(m u m^{-1}\right) d u d m \\
& =\int_{M} \delta_{M}(m)^{-1}(D f)^{-}\left(m^{-1} \cdot \gamma_{\rho}\right) d m \\
& =\int\left(\left|\alpha_{1}\right|^{-(n-1)} \cdots\left|\alpha_{n}\right|^{n-1}\right)^{-1}(D f)^{-}\left(m^{-1} \cdot \gamma_{\rho}\right) \prod_{1<i<j<n} d w_{i j} \frac{d \alpha_{1} \cdots d \alpha_{n}}{\left|\alpha_{1}\right|^{n} \cdots\left|\alpha_{n}\right|} \\
& =\int\left|\alpha_{1}\right|^{-1}\left|\alpha_{2}\right|^{-2} \cdots\left|\alpha_{n}\right|^{-n}(D f)^{\wedge}\left(m^{-1} \cdot \gamma_{\rho}\right) \prod_{1<i<j<n} d w_{i j} d \alpha_{1} \cdots d \alpha_{n} .
\end{aligned}
$$


We recall that

$$
m^{-1} \cdot \gamma_{\rho}={ }^{t} W \gamma_{\rho} W \quad \text { if } m=\left(\begin{array}{cc}
W & 0 \\
0 & { }^{t} W^{-1}
\end{array}\right) \in M
$$

In some further detail, we can write

$$
\begin{aligned}
m^{-1} \cdot \gamma_{\rho} & =\left(\begin{array}{ccc}
\alpha_{1} & & w_{i j} \\
& \ddots & \\
0 & & \alpha_{n}
\end{array}\right]\left[\begin{array}{ccc}
s\left(\rho_{n}\right) s\left(\rho_{n-1}\right)^{-1} & & 0 \\
& \ddots & \\
0 & & s\left(\rho_{1}\right)
\end{array}\right]\left[\begin{array}{ccc}
\alpha_{1} & & 0 \\
& \ddots & \\
w_{i j} & & \alpha_{n}
\end{array}\right] \\
& =\left(\begin{array}{cccc}
\alpha_{2}^{1} s\left(\rho_{n}\right) s\left(\rho_{n-1}\right)^{-1}+\cdots & w_{21} s\left(\rho_{n-1}\right) s\left(\rho_{n-2}\right)^{-1} \alpha_{2}+\cdots & \cdots & w_{n 1} s\left(\rho_{1}\right) \alpha_{n} \\
& \alpha_{2}^{2} s\left(\rho_{n-1}\right) s\left(\rho_{n-2}\right)^{-1}+\cdots & \cdots & w_{n 2} s\left(\rho_{1}\right) \alpha_{n} \\
& \ddots & & \vdots \\
& & & \alpha_{n}^{2} s\left(\rho_{1}\right)
\end{array}\right) .
\end{aligned}
$$

We observe that

$$
\Delta_{k}\left(m^{-1} \cdot \gamma_{\rho}\right)=\alpha_{n-k+1}^{2} \cdots \alpha_{n}^{2} s\left(\rho_{k}\right)
$$

Therefore

$$
\begin{aligned}
\operatorname{Tr} \pi(\rho)(D f) & =\int\left|\alpha_{1}\right|^{-1} \cdots\left|\alpha_{n}\right|^{-n} \prod_{k=1}^{n}\left|\Delta_{k}\left(m^{-1} \cdot \gamma_{\rho}\right)\right| \hat{f}\left(m^{-1} \cdot \gamma_{\rho}\right) \\
& \times \prod_{1<i<j<n} d w_{i j} d \alpha_{1} \cdots d \alpha_{n} \\
& =\int\left|\alpha_{1}\right| \cdots\left|\alpha_{n}\right|^{n}\left|s\left(\rho_{1}\right) \cdots s\left(\rho_{n}\right)\right| \hat{f}\left(m^{-1} \cdot \gamma_{\rho}\right) \prod_{1<i<j<n} d w_{i j} d \alpha_{1} \cdots d \alpha_{n} .
\end{aligned}
$$

Transforming out the coefficients of the $w_{i j}$ we obtain

$$
\begin{aligned}
& \operatorname{Tr} \pi(\rho)(D f)=\int\left|\alpha_{1} \cdots \alpha_{n}\right|\left|s\left(\rho_{n}\right)\right| \\
& \quad \times \hat{f}\left(\begin{array}{cccc}
\alpha_{1}^{2} s\left(\rho_{n}\right) s\left(\rho_{n-1}\right)^{-1}+\cdots & & & \\
& \alpha_{2}^{2} s\left(\rho_{n-1}\right) s\left(\rho_{n-2}\right)^{-1}+\cdots & & \\
w_{i j} & & \ddots & \alpha_{n}^{2}\left(\rho_{1}\right)
\end{array}\right) \prod_{i<j} d w_{i j} d \alpha_{1} \cdots d \alpha_{n}
\end{aligned}
$$

where in each diagonal entry $\alpha_{i}^{2} s\left(\rho_{n-i+1}\right) s\left(\rho_{n-i}\right)^{-1}+\cdots$, the expressions that appear in the sum involve only $\alpha_{j}, j>i$, and $s\left(\rho_{j}\right), j<n-i+1$. Then, successively letting $x_{1}=\alpha_{1}^{2} s\left(\rho_{n}\right), x_{2}=\alpha_{2}^{2} s\left(\rho_{n-1}\right), \ldots$, we see that 


$$
\begin{aligned}
& \int_{R_{F}^{n}} \operatorname{Tr} \pi(\rho)(D f) d \rho=\int_{R_{F}^{n-1}} \int\left|\alpha_{2} \cdots \alpha_{n}\right| \\
& \times \hat{f}\left(\begin{array}{ccc}
x_{1} s\left(\rho_{n-1}\right)^{-1}+\cdots & & w_{i j} \\
& \ddots & \\
w_{i j} & & \alpha_{n}^{2} s\left(\rho_{1}\right)
\end{array}\right) \prod_{i<j} d w_{i j} d x_{1} d \alpha_{2} \cdots d \alpha_{n} d \rho \\
& =\int_{R_{F}^{n-1}}\left|\alpha_{2} \cdots \alpha_{n}\right|\left|s\left(\rho_{n-1}\right)\right| \\
& \times \hat{f}\left(\begin{array}{llll}
x_{1} & & & w_{i j} \\
& \alpha_{2}^{2} s\left(\rho_{n-1}\right) s\left(\rho_{n-2}\right)^{-1}+\cdots & & \\
& & \ddots & \\
w_{i j} & & \alpha_{n}^{2} s\left(\rho_{1}\right)
\end{array}\right) \prod_{i<j} d w_{i j} d x_{1} d \alpha_{2} \cdots d \alpha_{n} d \rho \\
& =\int \hat{f}\left(\begin{array}{lll}
x_{1} & & w_{i j} \\
& \ddots & \\
w_{i j} & & x_{n}
\end{array}\right) \prod_{i<j} d w_{i j} d x_{1} \cdots d x_{n}=f(0)=f\left(1_{G}\right) .
\end{aligned}
$$

This completes the proof.

5. Concluding remarks. Work carried out thus far on the Plancherel formula for parabolic subgroups $P=M A N$ of semisimple (reductive) Lie groups has revealed two very distinct types of behavior. To explain, let $P_{0}$ be the kernel of the modular function $\delta_{p}$ of $P$. Calvin Moore has proven that the quotient space $\hat{N} / P$ is measure-theoretically finite, i.e. there are a finite number of $P$-orbits of positive Plancherel measure whose union is conull. The distinct behavior mentioned above corresponds to whether $\hat{N} / P_{0}$ is measure-theoretically finite or not. Thus far, substantial results have only been obtained in the latter case. In that situation, the Dixmier-Pukanszky operators arise from semi-invariants that live on $N$; in fact in many instances, on a normal abelian subgroup of $N$. Abelian Fourier analysis can then be brought to bear on the matter. But in the contrary case $\left(\hat{N} / P_{0}\right.$ meas.-theor. finite), the Dixmier-Pukanszky operator will likely live on all of $P_{0}$ and very little (aside from several low-dimensional groups) is known.

The author and $\mathbf{J}$. Wolf have developed a theory which gives necessary and sufficient conditions for $\hat{N} / P_{0}$ to not be meas.-theor. finite; and in that case locates the semi-invariant in the center of the enveloping algebra of the nilradical. Borel groups are always in that category. But it is only in the $A_{n}$ and $C_{n}$ cases that the center of the enveloping algebra lies inside the enveloping algebra of an abelian ideal. This accounts for our ability to treat the semi-invariant by abelian Fourier analysis. In the $B_{n}$ and $D_{n}$ cases, we expect that the semi-invariants must live on nilpotent, nonabelian subgroups of $N$. Hence the analysis of $\$ 3$ (specifically Proposition 3.2) must be replaced by a nilpotent Fourier analysis that will rely heavily on the Kirillov orbit method. Such work is in progress.

Two final remarks. (a) In previous work on the subject, all parabolics in which the Plancherel formula has been computed have enjoyed the property that their nilradicals have square-integrable representations mod the center. This is not the 
case for the Borel groups in $\S 4$, nor for that matter for the Borel subgroups of $\operatorname{SL}(n, \mathbf{F})$.

(b) In the Lie group case $(\mathbf{F}=\mathbf{R})$, the Dixmier-Pukanszky operator for a parabolic $P$ inside a semisimple group $G$ will not change when we pass to the complexification $P_{c} \subseteq G_{c}$, i.e. in some sense it is enough to consider only split groups. In order to understand a general parabolic in the split case we must first deal with Borel subgroups, as we have in this paper.

\section{REFERENCES}

1. I. Gelfand, M. Graev and I. Piatetski-Shapiro, Representation theory and automorphic functions, Saunders, Philadelphia, 1969.

2. F. Keene, R. Lipsman and J. Wolf, The Plancherel Formula for parabolic subgroups, Israel J. Math. 28 (1977), 68-90.

3. A. Kleppner and R. Lipsman, The Plancherel Formula for group extensions, Ann. Sci. École Norm. Sup. 5 (1972), 459-516.

4. 103-132.

5. R. Lipsman and J. Wolf, The Plancherel Formula for parabolic subgroups of the classical groups, J. Analyse Math. 34 (1978), 120-161.

6. A. Weil, Basic number theory, Springer-Verlag, New York, 1974.

7. J. Wolf, Classification and Fourier inversion for parabolic subgroups with square integrable nilradical, Mem. Amer. Math. Soc. No. 225 (1979).

Department of Mathematics, University of Maryland, College Park, Maryland 20742 